\title{
A SHORT PROOF OF FAN'S FIXED POINT THEOREM
}

\author{
FRODE TERKELSEN
}

\begin{abstract}
Fan's fixed point theorem for multivalued functions in locally convex spaces is proved by means of Brouwer's fixed point theorem and the concept of a partition of unity.
\end{abstract}

Let $X$ and $Y$ be topological spaces, and let $2^{Y}$ denote the set of subsets of $Y$. A function $T: X \rightarrow 2^{Y}$ (called a multivalued function of $X$ into $Y$ ) is upper semicontinuous if for each $x_{0} \in X$ and each neighborhood $W$ of $T\left(x_{0}\right)$ in $Y$, there exists a neighborhood $V$ of $x_{0}$ in $X$ such that $x \in V$ implies $T(x) \subset W$. We shall give a short proof of Fan's fixed point theorem for multivalued functions, modifying a method of Browder [1].

THEOREM (K. FAN [2]). Let $K$ be a nonempty compact convex subset of a Hausdorff locally convex topological vector space $E$, and let $T: K \rightarrow 2^{K}$ be an upper semicontinuous function such that $T(x)$ is nonempty closed and convex for each $x \in K$. Then there exists $x_{0} \in K$ with $x_{0} \in T\left(x_{0}\right)$.

Proof. Let $\left\{U_{i}: i \in I\right\}$ denote a neighborhood base at 0 in $E$ consisting of open convex circled sets. For each $i \in I$ there exists a finite set $\left\{x_{i j}: j \in J(i)\right\} \subset K$ with $K \subset \bigcup_{j_{\in} J(i)}\left(x_{i j}+U_{i}\right)$. There exists a continuous partition of unity subordinate to this covering, i.e. for $j \in J(i)$ there are continuous functions $\alpha_{i j}: K \rightarrow R$ with $\alpha_{i j}(x) \geqq 0$ for $x \in K, \alpha_{i j}(x)=0$ for $x \notin x_{i j}+U_{i}$, and $\sum_{j \in J(i)} \alpha_{i j}(x)=1$ for $x \in K$. Choose $y_{i j} \in T\left(x_{i j}\right)$ arbitrarily, and define the function $f_{i}: K \rightarrow E$ by $f_{i}(x)=\sum_{j \in J(i)} \alpha_{i j}(x) y_{i j}$. The convex hull $C_{i}$ of $\left\{y_{i j}: j \in J(i)\right\}$ is homeomorphic to a Euclidean ball, with $C_{i} \subset K$ and $f_{i}\left(C_{i}\right) \subset C_{i}$. By Brouwer's fixed point theorem we may choose $x_{i} \in C_{i}$ such that $f_{i}\left(x_{i}\right)=x_{i}$.

The neighborhood base $\left\{U_{i}: i \in I\right\}$ is directed by $\supset$. Let $x_{0} \in K$ be a cluster point of the corresponding net $\left\{x_{i}: i \in I\right\}$ in $K$, and suppose $x_{0} \notin$ $T\left(x_{0}\right)$. By separation there is a closed convex neighborhood $W$ of $T\left(x_{0}\right)$ with $x_{0} \notin W$. Since $T$ is upper semicontinuous there exists a neighborhood $V$ of $x_{0}$ with $V \cap W=\varnothing$ such that $x \in V \cap K$ implies $T(x) \subset W$. Choose $m \in I$ with $U_{m}+U_{m} \subset V-x_{0}$. There exists an $i \in I$ with $U_{m} \supset U_{i}$ such that $x_{i} \in x_{0}+U_{m}$, and then $x_{i}+U_{i} \subset V$ holds. For any $j \in J(i)$ with $\alpha_{i j}\left(x_{i}\right) \neq 0$

AMS (MOS) subject classifications (1970). Primary 54C60, 47H10. 
we have $x_{i} \in x_{i j}+U_{i}$, hence $x_{i j} \in V$ which implies $y_{i j} \in W$. Then

$$
x_{i}=f_{i}\left(x_{i}\right)=\sum_{j \in J(i)} \alpha_{i j}\left(x_{i}\right) y_{i j} \in W,
$$

contradicting that $x_{i} \in V$. Therefore $x_{0} \in T\left(x_{0}\right)$ holds.

Fan's fixed point theorem immediately implies the theorems of Kakutani [3] $\left(E=R^{\prime \prime}\right)$, Tychonoff [4] $(T(x)=\{f(x)\}$ for a continuous function $f: K \rightarrow K)$, as well as the fundamental Brouwer fixed point theorem used in the proof above.

\section{REFERENCES}

1. F. E. Browder, The fixed point theory of multi-valued mappings in topological vector spaces, Math. Ann. 177 (1968), 283-301. MR 37 \#4679.

2. K. Fan, Fixed-point and minimax theorems in locally convex topological linear spaces, Proc. Nat. Acad. Sci. U.S.A. 38 (1952), 121-126. MR 13, 858.

3. S. Kakutani, A generalization of Brouwer's fixed point theorem, Duke Math. J. 8 (1941), 457-459. MR 3, 60.

4. A. Tychonoff, Ein Fixpunktsatz, Math. Ann. 111 (1935), 767-776.

Institute of Economics, University of Copenhagen, Copenhagen, Denmark 